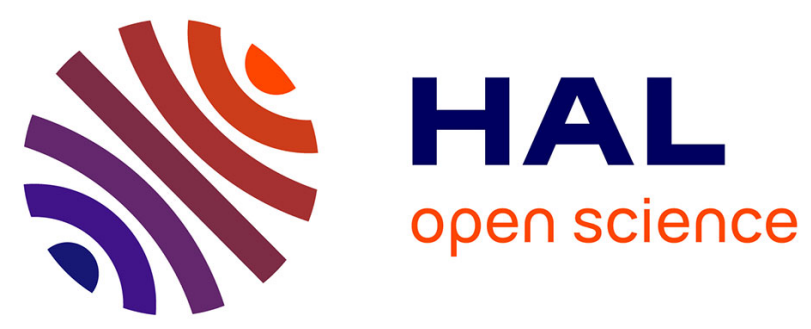

\title{
SEAD: A simple and efficient adaptive data dissemination protocol in vehicular ad-hoc networks
}

Imen Achour, Tarek Bejaoui, Anthony Busson, Sami Tabbane

\section{To cite this version:}

Imen Achour, Tarek Bejaoui, Anthony Busson, Sami Tabbane. SEAD: A simple and efficient adaptive data dissemination protocol in vehicular ad-hoc networks. Wireless Networks, 2015, pp.11. 10.1007/s11276-015-1050-9 . hal-01242289

\section{HAL Id: hal-01242289 \\ https://inria.hal.science/hal-01242289}

Submitted on 11 Jan 2016

HAL is a multi-disciplinary open access archive for the deposit and dissemination of scientific research documents, whether they are published or not. The documents may come from teaching and research institutions in France or abroad, or from public or private research centers.
L'archive ouverte pluridisciplinaire HAL, est destinée au dépôt et à la diffusion de documents scientifiques de niveau recherche, publiés ou non, émanant des établissements d'enseignement et de recherche français ou étrangers, des laboratoires publics ou privés. 


\title{
SEAD: A Simple and Efficient Adaptive Data Dissemination Protocol in Vehicular Ad-hoc Networks
}

\author{
Imen Achour - Tarek Bejaoui • Anthony Busson - Sami Tabbane
}

Received: date / Accepted: date

\begin{abstract}
Vehicular Ad-Hoc Network (VANET) is becoming a promising technology for improving the efficiency and the safety of Intelligent Transportation Systems (ITS) by deploying a wide variety of applications. Smart vehicles are expected to continuously exchange a huge amount of data either through safety or non-safety messages dedicated for road safety or infotainment and passenger comfort applications, respectively. One of the main challenges posed by the study of VANET is the data dissemination design by which messages have to be efficiently disseminated in a high vehicular speed, intermittent connectivity, and highly dynamic topology. In particular, broadcast mechanism should guarantee fast and reliable data delivery within a limited wireless bandwidth in order to fit the real time applications' requirements. In this work, we propose a Simple and Efficient Adaptive data Dissemination protocol called "SEAD". On the one hand, the originality of this work lies in its simplicity and efficiency regardless the application's type. Simplicity is achieved through a beaconless strategy adopted to take into account the surrounding vehicles' density. Thanks to a metric locally
\end{abstract}

\section{Achour}

Mediatron Lab. SupCom, University of Carthage, Tunisia

Telnet Innovation Labs. Telnet Holding

E-mail: imen.achour@supcom.tn

T. Bejaoui

Mediatron Lab. SupCom, University of Carthage, Tunisia

E-mail: Tarek.bejaoui@supcom.tn

\section{A. Busson}

Laboratory of Parallel Computing

University of Lyon 1 - ENS - CNRS - Inria, France

E-mail: anthony.busson@inria.fr

S. Tabbane

Mediatron Lab. SupCom, University of Carthage, Tunisia

E-mail: sami.tabbane@supcom.rnu.tn measured, each vehicle is able to dynamically define an appropriate probability of rebroadcast to mitigate the broadcast storm problem. Efficiency is manifested by reducing excessive retransmitted messages and hence promoting the network capacity and the transmission delay. The simulation results show that the proposed protocol offers very low packet drop ratio and network load while still maintaining a low end-to-end delay and a high packet delivery. On the other hand, SEAD presents a robust data dissemination mecanism which is suitable either for safety applications or for other kinds of application. This mechanism is able to adapt the protocol performance in terms of packet delivery ratio to the application's requirements.

Keywords Vehicular Ad hoc Networks · Data dissemination - Vehicle to Vehicle Communication • Vehicles' Density · Redundancy and Adaptive protocol

\section{Introduction}

With the advent of emerging intelligent transportation systems (ITS), tremendous efforts have been put on enhancing the safety and the efficiency of transportation services by developing original applications. In particular, Vehicular Ad-hoc Networks (VANETs) are considered as the most prominent technologies for ensuring and maintaining a wide variety of applications ranging from road safety and traffic management to infotainment and advertising applications.

Composed of smart and connected vehicles, VANETs are self-organizing wireless networks. Vehicles move only on predetermined roads, and they do not have the problem of resources limitation in terms of data storage and power. Yet, vehicular networks have several challenging characteristics that distinguish them from the other 
mobile ad hoc networks (MANET). The mobility of vehicles under different traffic conditions, as for instance during traffic jams, accidents, traffic lights, rush hours, etc, results in a highly dynamic change in the network topology. Furthermore, the high mobility property of nodes (vehicles) leads to an intermittent connectivity. Thus the connection link between two vehicles may frequently disappear while they are exchanging data. Such particular features often bring new challenges in wireless technologies and networking.

The appearance of IEEE 802.11p standard [9] specifically designed for $\mathrm{V} 2 \mathrm{~V}$ communication and according to the inter-vehicle communication requirements constitutes an important step. However, further studies on data dissemination should be done on the next step.

Moreover, vehicular networks are suffering from limited resource bandwidth. Indeed, according to the Federal Communications Commission (FCC), the allocated band in USA is divided into seven channels with 10 $\mathrm{MHz}$ in bandwidth: 1 control channel $(\mathrm{CCH})$ and 6 service channels ( $\mathrm{SCH}$ ). Only $\mathrm{CCH}$ is dedicated to deliver safety messages (e.g., Emergency electronic brake lights Messages, Collision Risk Warning, Traffic hazard warnings, etc.) which are considered the most valuable applications family since they meant to address passenger safety. The broadcast of such type of messages is a critical issue in vehicular network. They need to be effectively exchanged among vehicles by achieving a high packet delivery within a certain time limit and an acceptable overhead. However, the main scope of ITS is not only to provide safety services, but also to make the best utilization of available bandwidth in providing entertainment services.

On the bases of these constraints specific data dissemination protocols should be designed to fit the different requirements of the various VANETs' applications [7] [8] [33] [20]. Traditional broadcasting techniques, such as blind flooding, seriously suffer from the so called Broadcast Storm Problem [26]. Indeed, each vehicle must immediately rebroadcast every received message in order to ensure the data delivery for distant vehicles situated in the area of interest. Thereby, an excessive number of redundant messages is transmitted leading to a large amount of bandwidth consumption. This problem is getting more serious when the network density is high. This results in a high channel contention and a large number of collisions [12].

Several research activities addressing data broadcast algorithms propose new strategies to cope with this problem. The most common way by which these strategies perform is that only a set of selected vehicles will be relaying nodes for the purpose of reducing the number of retransmissions. However, the difference between these strategies lies in how the vehicles are selected as relay nodes and according to which criteria? Based on some reviews [10][19][5] we may essentially distinguish three basic categories, namely delay-based techniques, probability-based techniques and hybrid techniques. The different criterion involved in the relaying node selection are basically the distance between the transmitter and the receiver, the message direction with relation to the vehicle moving direction and the surrounding vehicles' density.

In this context, we propose an original hybrid (delay and probability) broadcast protocol named "SEAD". The contribution of this work is twofold in providing an efficient data broadcast protocol and in offering the ability of adapting the protocol performance in accordance with the application's requirements. The proposed protocol is efficient since it takes into account three significant parameters for the data broadcast decision, namely the distance, the traffic density and the message direction. The efficiency of SEAD not only refers to a high packet delivery ratio and an acceptable end to end delay but also to a significant reduce in bandwidth consumption. Added to that, SEAD is an adaptive data broadcast protocol that may behave efficiently either for safety or non-safety messages exchange. Thanks to a specific defined parameter, we are able to accurately adjust the packet delivery ratio according to the application's needs.

The reminder of this paper is organized as follows. In Section 2, we report previous works on data broadcast protocols. Section 2 provides a detailed description of SEAD protocol. Section 4, presents the simulation environment and discuss the performance evaluation. Finally, concluding remarks and future works are presented in Section 5.

\section{Related Works}

Recently, a set of techniques were proposed to address the data dissemination issue in vehicular environment upon three basic transmission strategies, namely unicast, multicast and broadcast [32] [13] [24] [11] [30] [15] [16] [17]. These techniques were surveyed in different works [10] [19] [5] [28]. Some of them are specifically designed for the vehicle to infrastructure communication (V2I) paradigm, whereas, some others are dedicated for a V2V communication. The V2V data broadcast is considered the most relevant technique for critical message dissemination. However, in dense network, this technique is facing the so-called "Broadcast storm Problem" due to an excessive amount of data exchanged within scarce wireless resources.

To cope with this problem, researchers have proposed 


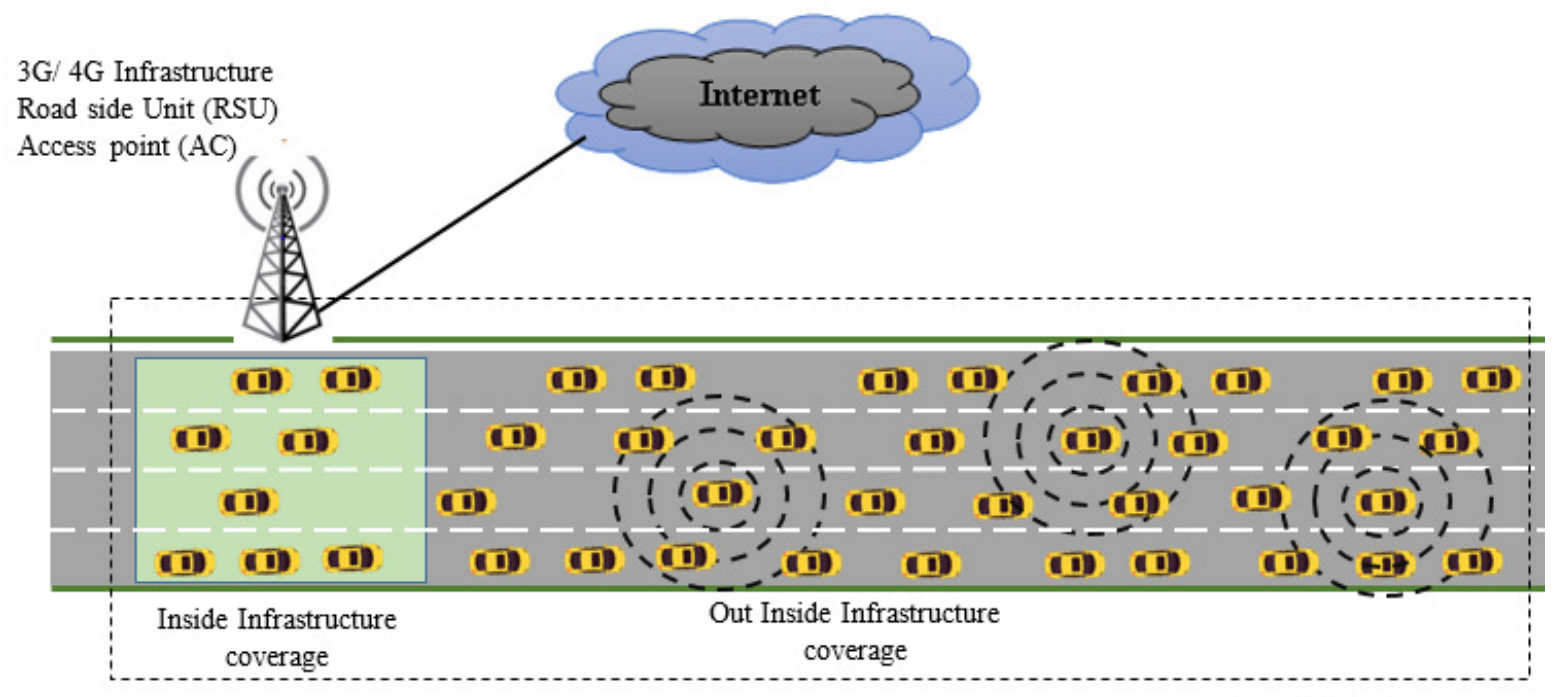

Area of Interest

Fig. 1 System Architecture

various suppression techniques. In particular, authors in [29] have proposed three basics Network Layer suppression strategies: a delay based strategy called "slotted 1-persistence", a probabilistic based strategy denoted "weighted p-persistence, and a hybrid scheme "slotted p-persistence". On the bases of simulation results, [29] demonstrates that "slotted 1-persistence" disseminating protocol (S1PD) shows better performance compared to the two other protocols. S1PD yields a significant decrease in the number of redundant rebroadcast whilst ensuring a low end-to-end delay and a high packet delivery. The idea of S1PD is to divide the transmission range of a sender throughout the road into a fixed number of segments, denoted "slots". Each segment is then associated to a specific timer value, denoted "time slot". The shortest time slot will be assigned to the farthest segment. Thereby, receiving vehicles belonging to the farthest sender's segment will be given the shortest waiting time to rebroadcast. The vehicle's timer is started upon the reception of a new message. When the timer expires, the vehicle forwards the message if it has not received any duplicates during the waiting time; otherwise, the packet is discarded. Hence, vehicles belonging to other segments would have sufficient time to cancel their transmissions once they receive a duplicated message. As a result, redundant broadcasts may be suppressed.

However, "Slotted1-Persistence" technique may suffer from a Timeslot Boundary Synchronization problem, as its denoted in [4]. This problem can occur when multiple vehicles belonging to the same segment and assigned to the same timer start their transmissions simultaneously, which may result in a high number of collisions. Towards solving this problem, different optimization works in S1PD protocol have been proposed. Authors in [21] denote that both link and network layer should be specifically designed to address the synchronization problem. To this end, an extra micro delay must be added to the timer in the network layer. Also a pseudo-random delay must be added to SIFS in the IEEE 802.11p MAC layer. The time slot assignment, in [21], is carried out according to the distance of the receiver vehicle from the transmitter and to its moving direction toward the broadcast message direction.

Furthermore, in order to more efficiently alleviate these problems, some recent works $[22,23,6,27,3]$ have involved the surrounding vehicles' density in their broadcast protocol design. Based on the vehicles' density knowledge within the transmission range, forwarding decision is becoming smarter leading to an effective control on the transmission redundancy and hence a substantial improve in the protocol performance.

Unlike our scheme, all the proposed density based protocols relay on a periodic exchange of beacon messages for density estimation. Gathering the information contained in beacons (vehicle's location, speed, etc.) enables each vehicle to continuously maintain and update tables on the neighbors' position information. Nevertheless, in dense and highly dynamic networks, the management of these tables is becoming a hard task for vehicles' density estimation.

On the other hand, some researchers have addressed the efficiency of broadcasting protocol by saving the network resources consumption while maintaining a high data broadcast performance. To this end, a new trend of protocols, based on the network coding technique 
(NC) [2] [31] is appeared. $\mathrm{NC}$ has recently caught the attentions of many researchers in the field of wireless communications in vehicular environment. The main idea of this technique is to enable intermediate nodes to combine different received packets before forwarding, instead of sending them separately. The various NC dissemination protocols proposed in the literature were reviewed and classified in [1]. This technique has shown a significant improvement in data transmission efficiency with respect to the network throughput, wireless resources capacity, energy consumption and reliability issues. Although these salient properties, NC technique have to face a complicated negotiation. On one hand, "Which packets to transmit ?" that means how many packets and what is the best combination of packets that should be mixed together before forwarding in order to achieve a high packet delivery. On the other hand "When to transmit?" which means how much time incoming packets should wait before generating a new coded packet. Indeed, according to $\mathrm{NC}$ technique a node is allowed to combine only successfully decoded packets. However, the success of this latter procedure relatively depends on the received packets. So, the forwarding node have to wait the reception of a certain number of native packets in order to decode the new received packet.

Our work further differs from these protocols, in a focus on increasing the dissemination reliability while effectively reducing the network bandwidth utilization through a simple design. In particular, thanks to the defined redundancy ratio metric, vehicles transmitters are able to dynamically adjust the probability of rebroadcast according to the surrounding vehicles' density without the need of neighbors' management. Added to that, by simply tuning one parameter, our protocol may be accurately adapted to specific application's requirements while controlling the packet delivery ratio and hence the redundant transmissions.

\section{Simple and Efficient Adaptive Data Dissemination Protocol}

In this paper we propose an original data dissemination protocol called "Simple and Efficient Adaptive Data Dissemination Protocol - SEAD". SEAD is a hybrid protocol which combines delay and probability-based dissemination schemes. The aim of this protocol is to be at once effective and simple. For the first purpose, SEAD tackles the broadcast storm problem by reducing excessive broadcasts while offering high packet delivery and low end-to-end delay in a highway environment. Thus, in addition to the distance parameter used for computing the waiting time, SEAD takes into account the vehicles' density and also the messages' direction to determine its re-broadcast probability. On the other side, SEAD applies a simple design through which no beacon exchange containing neighbors' positions, speed and direction is required even though the vehicles' density is considered. Added to that, the key feature of SEAD is that it is a generic protocol which may suits all types of application. Indeed, the packet delivery ratio is handled according to the application's requirements by simply fixing an appropriate value of a parameter, denoted " $\alpha$ " throughout the rest of this paper.

To further understand the protocol details, we first propose to describe general assumptions and requirements for a proper operation. Next, we investigate the evolution of the "redundancy ratio" metric under various vehicles' density. And then, a thorough description of the basic steps of SEAD will be presented.

\subsection{Assumptions and requirements}

In this work, we mainly consider V2V communications, however a V2I communication may be involved for message generation as it is presented in Fig. 1. In this context, we assume that each vehicle is equipped with onboard wireless devices in compliance with the available IEEE 802.11p standard. Besides, the proposed scheme assumes that every vehicle is able to permanently determine its current geographical position using Global Positioning System (GPS) or any other localization service [14]. Furthermore, we assume either the presence of a local application running on the source vehicle or the presence of a fixed infrastructure (Access point, Road side Unit, etc.) responsible for data message generation. All generated messages need to be disseminated within an area of interest. Throughout the article, we will often simply refer to them as messages or data packets.

\subsection{Redundancy Metric}

We refer to Redundancy Ratio " $r$ " as the metric responsible for measuring the number of received messages per new message. This metric is calculated as follows:

$$
r=\frac{\text { TotalReceivedMessages }(\text { original }+ \text { duplicated })}{\text { TotalNewMessages(original })}
$$

In our design, we assume that each vehicle is able to continuously updated its redundancy metric upon the receipt of a message. The reset of this metric is triggered when no packet is received during a predefined period of time $\Delta \mathrm{t}$. Given a fixed number of source vehicles, we 


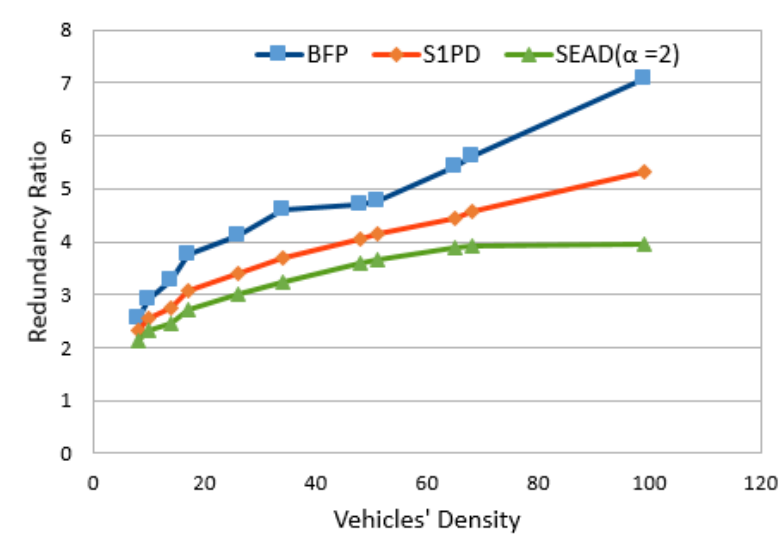

Fig. 2 Redundancy Ratio variation with relation to vehicles' density

have performed a set of simulations (for which detail will be described in Section 4) in order to study the impact of the vehicles' density on the redundancy ratio variation. The value of $\alpha$ parameter was set to 2 . Fig. 2 shows that the redundancy ratio inherently increases with the increase of the vehicles' density. This means that the number of redundant packets increases when the vehicles' density is becoming higher.

Hence, from this observation we conclude that the probability of rebroadcast in SEAD should be inversely proportional to the redundancy ratio metric. Then, higher the redundancy ratio, smaller the broadcasting probability is. This leads to reduce the probability of broadcast when the vehicles' density increases.

\subsection{SEAD Protocol Description}

The main concept of SEAD is presented by two flowdiagrams depicted in Fig. 3 and Fig. 4 that can be described as follows:

In our protocol, each message is identified by a unique ID which consists of the source vehicle's ID and the local packet ID. We assume that the packet's header contains the broadcasting node ID and its GPS coordinates. As well, each vehicle has a data buffer that stores the original data packets (not duplicated ones), either received or generated by the local application running on the transmitter vehicle. The SEAD protocol basically lies on two checking phases. Upon receiving a packet, the vehicle checks first whether the message's ID is already stored in the data buffer or not. This is performed in order to verify the newness of the message. If the message is not new, this means that the received message is redundant and should be discarded after updating the redundancy ratio "r" parameter. Otherwise, it is copied in the buffer and a second check procedure is triggered. The purpose behind this procedure is to

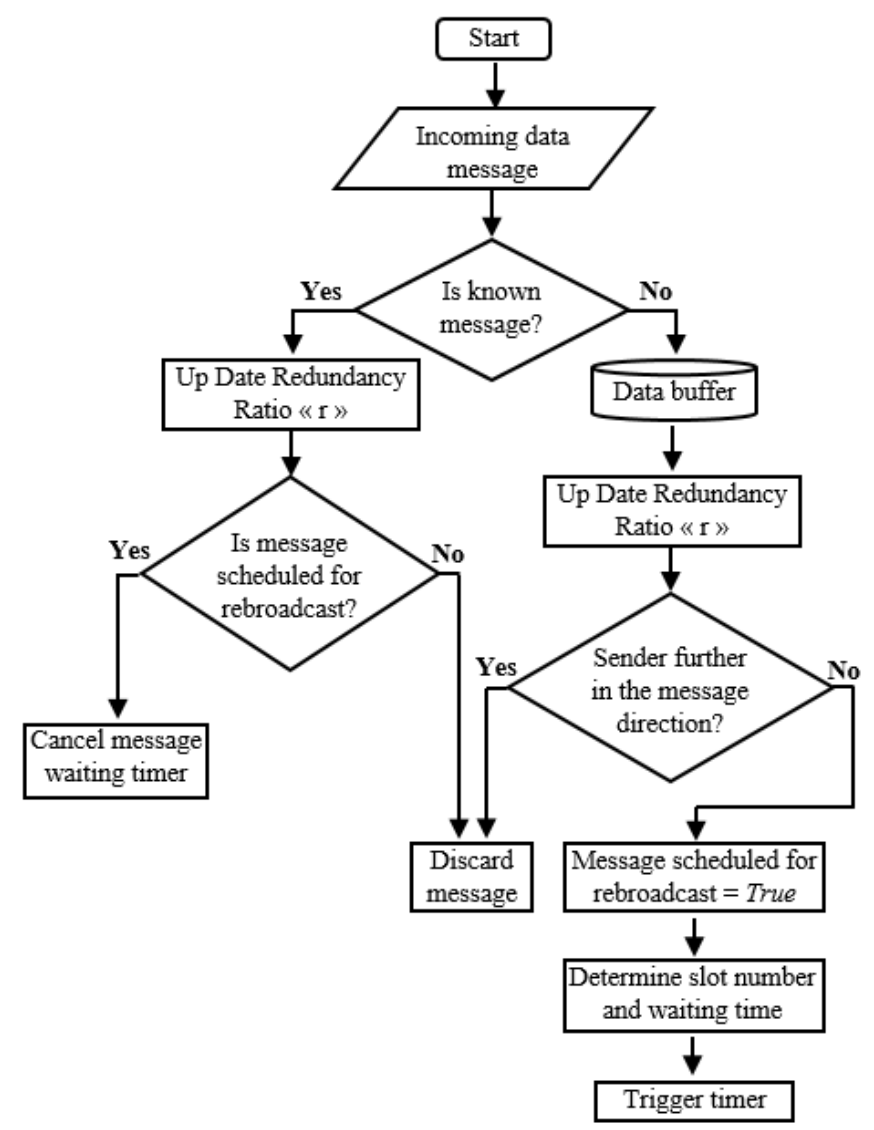

Fig. 3 Reception procedure of SEAD Protocol

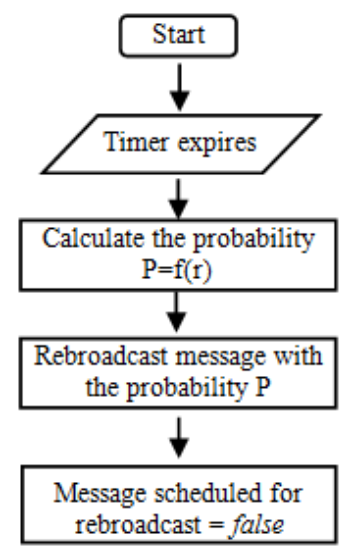

Fig. 4 Rebroadcasting procedure of SEAD Protocol

determine the relevance of the message regarding the receiver vehicle. Only received messages coming from vehicles in front are treated as relevant information that need to be further disseminated in the message direction (i.e. further behind the receiver vehicle). According to SEAD protocol, these messages must be tagged by the label "scheduled for rebroadcast = true". However, messages coming from vehicles behind are simply considered as an implicit acknowledgement about 
their reachability. Such messages are tagged by the label "scheduled for rebroadcast $=$ false" to prevent unnecessary retransmissions since they have already reached distant vehicles in the message direction. Each message labeled with "scheduled for rebroadcast = true" is then considered for a potential rebroadcast to which a waiting timer " $W_{t}$ " is assigned and immediately triggered. When the message's timer expires and no redundant message is received from other forwarders during the waiting process, the message is broadcast with a broadcast probability "P". Otherwise, the rebroadcast decision is cancelled.

The waiting time calculation adopted in SEAD, is inspired from S1PD scheme since it achieves the best performance among the other schemes proposed in [29]. Given a fixed number of slots " $N_{t}$ ", the waiting time is calculated as presented in equation (2):

$W_{t}=\left\lfloor N_{t} *\left(1-\frac{\min \left(D_{i j}, R\right)}{R}\right)\right\rfloor * \delta$

where $D_{i j}$ is the relative distance between the transmitter "i" and the receiver " $\mathrm{j}$ ", $\mathrm{R}$ is the average transmission range and $\delta$ is larger than one hop delay including medium access delay and propagation delay.

In attempt to further explain the adopted strategy, we

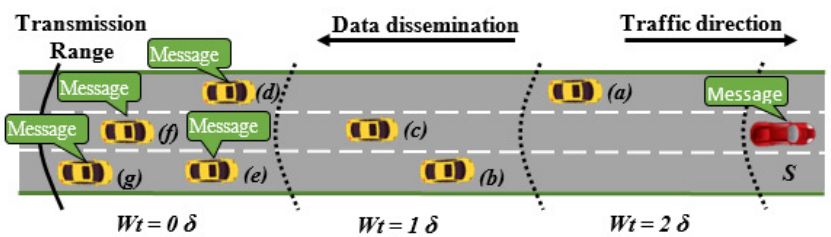

(a)

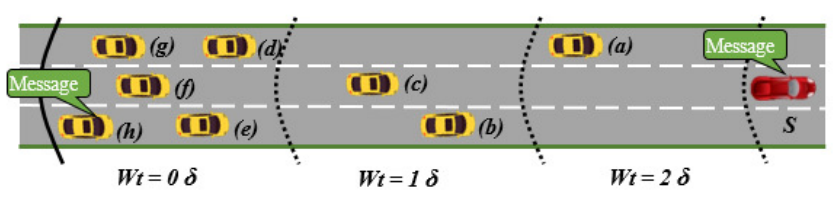

(b)

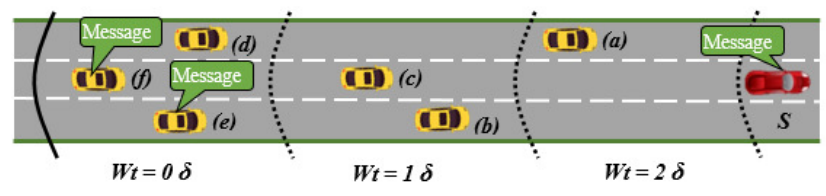

(c)

Fig. 5 Example of the delay based mechanism

propose a simple example presented in Fig. 5. Given a source node $(\mathrm{S})$ configured to reach about $300 \mathrm{~m}$ in its transmission range. All vehicles within its coverage and belonging to its area of interest are assigned to different road segments regarding their distances from the source vehicle. In our case, the number of segments (slots) is 3, i.e. one segment each $100 \mathrm{~m}$. According to equation (2), the waiting time duration is inversely proportional to the distance between receiving vehicles and source vehicle. Hence, as it is presented in Fig. 5-a, vehicles belonging to the farthest slot (Vehicles $\mathrm{d}, \mathrm{e}, \mathrm{f}$ and $\mathrm{g})$ are assigned to the minimum waiting delay $\left(W_{t}=\right.$ $0 \mathrm{~s})$. Vehicle (b) and vehicle (c) must wait $1 \delta$, whereas, vehicle (a) have to wait $2 \delta$ before its retransmission. The $\delta$ value is a sufficient period of time during which any vehicle have the time to receive retransmitted message from any other vehicle before its transmission. On the basis of this strategy, only vehicles belonging to last road segment will be the unique forwarders and not all receivers. Hence, assigning vehicles to different road segments with different timers will mitigate the broadcast storm by reducing the excessive number of redundant and simultaneous broadcasts. Nevertheless, the same problem can still occur in a small scale when huge vehicles belonging to the same segment are assigned to the same timer. Based on the same example, vehicles (d), (e), (f) and (g) will immediately send the received message at the same time, as Fig. 5-a shows. In this case, no collisions can occur thanks to the mechanism of backoff. However, when the number of vehicles belonging to the same segment is becoming higher, serious collisions will happen.

To cope with this problem we have proposed an original and simple way of calculating the re-broadcast probability "P". Thanks to this probability SEAD is capable to operate in-line without need of beacon exchange to consider the surrounding vehicles' density. This probability, given by equation (3), depends on the current redundancy ratio value " $r_{i}$ " calculated at the sending time "i" and the previous one " $r_{\text {prev }}$ " incorporated in the previous calculated probability "P $P_{\text {prev }}$ " for the last sent packet. We can notice, that the probability of broadcast is the product of all previous probabilities used in all previous transmissions. The main purpose of this correlation, is to continuously regulate the redundancy ratio in order to maintain a normalized amount of redundancy.

$$
\begin{aligned}
P_{i} & =\frac{2 \alpha}{r_{i}} \cdot P_{\text {prev }}=\frac{2 \alpha}{r_{0}} * \frac{2 \alpha}{r_{\text {prev }}} \\
& =\frac{2 \alpha}{r_{0}} \cdot \frac{2 \alpha}{r_{1}} \ldots \frac{2 \alpha}{r_{i-1}} \cdot \frac{2 \alpha}{r_{i}} \\
& =\frac{(2 \alpha)^{i+1}}{\prod_{k=0}^{i} r_{k}} .
\end{aligned}
$$

In this way, the forwarding probability is inversely proportional to the redundancy ratio and thus inversely proportional to the vehicles' density. Hence, regions with 
high density of vehicles will decrease the nodes' suitability to be a forwarding node. Yet, in low dense regions more candidates will be suitable for relaying received messages.

For the sake of simplicity, we resume the aforementioned example. As depicted in Fig. 5-b, only one vehicle (vehicle (h)) among five within the same segment has caught the opportunity of rebroadcast. However, two vehicles ((e) and (f)) among three were selected to reforward the received message in Fig. 5-c. This is due to the fact that lesser vehicles' density induces fewer redundancy amount and thereby increases the rebroadcast opportunity.

Furthermore, the adaptivity feature of SEAD protocol is expressed through a key parameter " $\alpha$ ". Via such parameter we are capable to tune the protocol behavior according to the application's performance requirements. This aspect is thoroughly studied in the next section.

\section{Performance Evaluation}

In this section we evaluate the performance efficiency of SEAD, carried out by means of extensive simulations in a vehicular environment. The simulation platform is constructed based upon ns-3 [18] simulator. Whereas, the realistic mobility trace in a highway environment is generated via a micro-traffic simulator named "SUMO - Simulation of Urban Mobility" [25] . For all simulations, we consider a straight three-lanes highway of $6 \mathrm{~km}$ length. We set the bit rate to $6 \mathrm{Mbit} / \mathrm{s}$ in the MAC layer. Assuming a Nakagami propagation model, we adjust the transmission power to achieve roughly 700 meters of transmission range. For the suppression technique mechanism, we set $\delta$ to $4 \mathrm{~ms}$ and we fix the total number of slots $N_{t}$ to 7 (one slot per $100 \mathrm{~m}$ ). For the application scenario, we configure the 5 first vehicles to generate at every second a new message of 500 bytes size. In order to evaluate the SEAD scalability, we vary the vehicles' density from 8 vehicles $/ \mathrm{km}$ to 99 vehicles $/ \mathrm{km}$ at the maximum speed of $20 \mathrm{~m} / \mathrm{s}$. Each plotted result is an average of 20 runs over $100 \mathrm{~s}$ with confidence interval of $95 \%$. All simulation parameters are summarized in Table 1.

Two disseminating protocols are picked for the performance comparison, namely:

- BFP (Blind Flooding Protocol): Is the most intuitive solution for data broadcast by immediately forwarding novel received packets.

- S1PD: Is a delay based technique [29] by which the packet transmission is only permitted at the expi-
Table 1 Simulation parameters

\begin{tabular}{ll}
\hline Parameters & Specifications \\
\hline Network simulator & ns-3.19 \\
Mobility trace generator & SUMO \\
Simulation duration & $100 \mathrm{~s}$ \\
Highway length (Unidirectional) & $6000 \mathrm{~m}$ \\
Vehicles' Density & 8 to 99 Vehicles $/ \mathrm{km}$ \\
Data packet frequency & $1 \mathrm{~Hz}$ \\
Data packet size & $500 \mathrm{bytes}$ \\
Number of source vehicles & 5 \\
Propagation model & $\mathrm{Nakagami}$ \\
Phy/Mac protocol & $\mathrm{IEEE} 802.11 \mathrm{p}$ \\
Bit rate & $6 \mathrm{Mbit} / \mathrm{s}$ \\
Transmission range & $\sim 700 \mathrm{~m}$ \\
$\delta$ & $4 \mathrm{~ms}$ \\
$N_{T}$ & 7 \\
Max speed & $20 \mathrm{~m} / \mathrm{s}$ \\
Number of run & 20 \\
\hline
\end{tabular}

ration of its assigned waiting time delay $W_{t}$. This is performed after checking the packet novelty.

For the performance evaluation we consider the following metrics:

- Packet Delivery Ratio (PDR): the average number of original packets successfully received by a vehicle, compared to the total number of generated messages.

- Forwarding Ratio (FR): the proportion of vehicles in the network that are involved in the rebroadcast of a source packet.

- End-to-End Delay (E2EDelay): the average difference between the generation time of a data packet by the source vehicle and the reception time of this packet by the last reached vehicle.

- Link Load (bit/s): the average of broadcast traffic (in terms of bits) received by each vehicle over a unit of time.

- Packet Drop Ratio (DROP): the average amount of erroneous received packets of a vehicle compared to the total received packets.

\subsection{Adaptive Robustness}

In this section we aim to study the impact of $\alpha$ parameter on the packet delivery ratio achieved while deploying the SEAD protocol. To this end, we plot in Fig. 6 the PDR in function of $\alpha$ ranging from 1 to 3. From this figure, we can clearly distinguish two basic parts of the curve: an increasing part for $\alpha$ value ranging from 1 to 1.9 then a steady state achieved from an $\alpha$ equal to 2 . The increasing part indicates the configurable phase of our proposed protocol. Hence, based on this curve we are capable to fix an accurate value of $\alpha$ in order to 


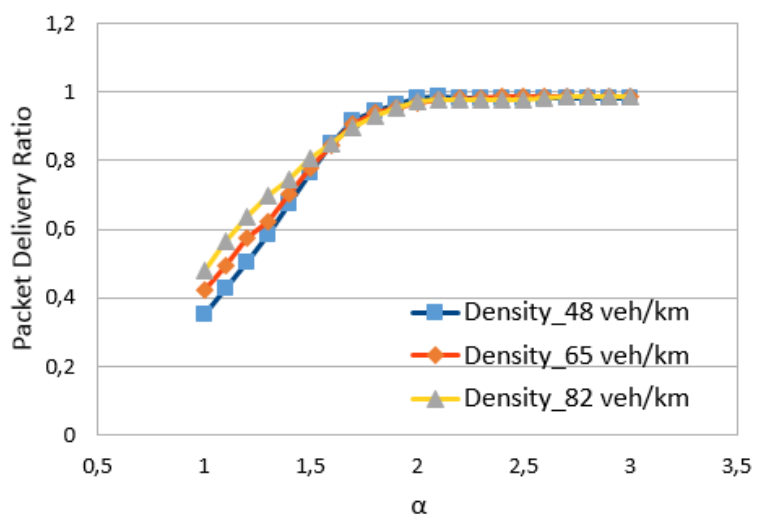

Fig. 6 Packet Delivery Ratio vs Various Alpha Value

maintain a particular amount of data reachability.

In fact, by tuning the $\alpha$ value, we are exactly setting the redundancy ratio, as it is presented in Fig. 7. This means that the $\alpha$ parameter allows as to fix a definite amount of redundancy which is enough to guaranty a certain ratio of packet delivery. Therefore, our protocol is adaptive while ensuring an efficient use of the limited bandwidth and the network capacity.

The choice of $\alpha$ value relatively depends on the deployed application and its performances' requirements. For example, applications relying on delivering advertisements and announcements are lesser critical than safety applications carrying emergency and traffic information. Thus, we can choose an $\alpha$ equal to 1.5 in order to reach roughly $80 \%$ of PDR for restaurants and hotel advertisement packets. Yet, we must choose an $\alpha$ equal to 2 to guaranty a high data reachability for accident warning messages.

Besides, under various vehicles' density, Fig. 6 shows that the PDR is still maintaining the same evolution with relation to $\alpha$ value variation. This observation is likewise the same for the redundancy ratio and the link load evolution, as depicted in Fig. 7 and Fig. 8, respectively. Hence, we can affirm the robustness of the adaptive aspect of our protocol.

Notice that the most significant applications in VANETs are those dedicated for ensuring the passenger's safety. That is why we have tuned the alpha value to 2 for the rest of our simulation in order to study the SEAD efficiency behavior toward safety message broadcast.

\subsection{Safety message dissemination efficiency}

On the basis of the simulation results presented in Fig. 9 , we can notice that the forwarding ratio drastically degrades with S1PD and SEAD, compared to BFP. In addition we can observe that the forwarding ratio degrades in S1PD and SEAD when the vehicles' density

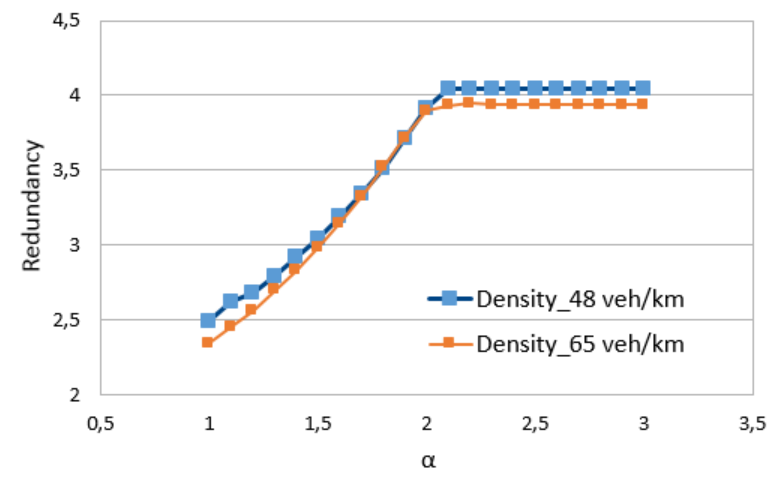

Fig. 7 Redundancy Ratio vs Various Alpha Value

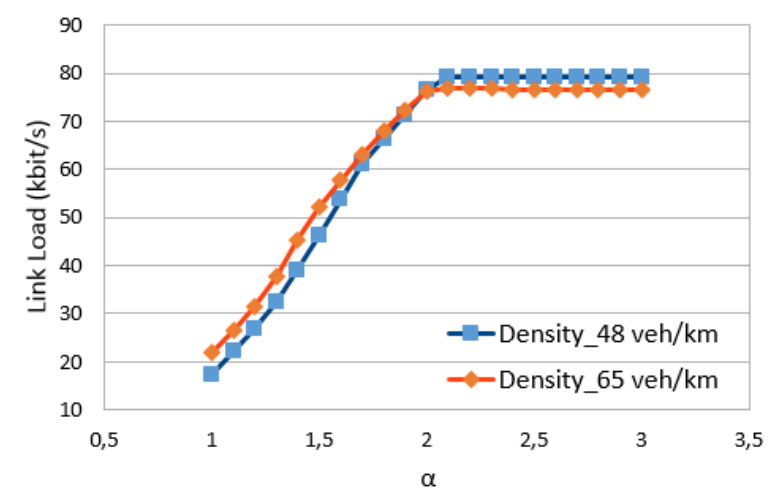

Fig. 8 Link Load vs Various Alpha Value

increases, while it rises in BFP. This shows the impact of the forwarding nodes selection on reducing unnecessary broadcasts. More the node selection is smarter, more the broadcast performance is better. However, SEAD shows the best performance since it is able to reduce by $50 \%$ the number of relaying nodes as compared to S1PD. As a result, SEAD is efficient in terms of reducing the number of packets hop, since less vehicles are involved in the re-forwarding process. Also, reducing the number of re-forwarders may have an important impact on the broadcast latency.

Moreover, Fig. 10 illustrates an important result of SEAD which is able to practically achieve the same PDR (as it is achieved by S1PD protocol) while reducing the number of forwarding nodes. Thereby, the network resources' consumption will significantly decreases. This observation is further proved through Fig. 11 that compares the link load for SEAD scheme with that obtained while using S1PD scheme. Here, SEAD scheme outperforms S1PD in terms of "link load" for all vehicles' densities. In particular, SEAD has reduced the link load about $80 \%$ compared to that of S1PD in high dense network, which illustrates the protocol scalability. This amounts to a substantial decline in the redundant message broadcast. Fig. 2 has shown that from a vehi- 
cles' density equal to 60 the redundancy ratio presents a threshold effect according to which " $\mathrm{r}$ " value does not exceed 4. This improvement, leads to save the limited bandwidth and to increase the network capacity in order to allow other types of applications to be run simultaneously.

Furthermore, SEAD presents in Fig. 12 a better drop ratio compared to S1PD. This highlights the inherent effect of SEAD protocol on enhancing the data broadcast reliability by reducing erroneous received messages. Thus, we can deduce how efficient SEAD protocol to alleviate the broadcast storm effect by decreasing the network contention and collisions compared to S1PD, while still achieving high PDR and lower end to end delay, as shown in Fig. 10 and Fig. 13. These properties confirm the protocol performance for real time applications, in particular, for safety and critical message dissemination.

Thanks to SEAD, each node will be able to adapt its suitability to be a re-broadcaster node according to three important factors: its distance from the source node, the current state of the network density and the direction from which it receives packets (from upstream or downstream). Thereby, each node will be able to get most likely the best decision without the need of information and feedback from neighboring nodes. As a result, the efficiency of SEAD emerges from the adaptive local behavior of each node, since each node is acting on its own.

On the other hand, the focus on SEAD on its own leads to deduce the following statement. On the bases of the defined probability in equation (3), SEAD protocol has significantly reduced the forwarding ratio and the network resources consumption (in terms of link load). In fact, the probability of broadcast is considered as a correction factor that attempts to continuously maintain a fix amount of redundancy even-though the vehicles' density increases.

Obviously excessive redundant packets may result in severe contention and collisions problems, nevertheless, a certain amount of redundancy should be preserved to guarantee a high packet delivery in a lossy network. Thus, a trade-off between the cost of unnecessary transmissions and the need of high reachability should be done according to the applications' requirements and the environmental conditions. In response to this fact, we have designed our protocol to be more flexible and offering the possibility to handle the required ratio of packet delivery. Thereby, we are able to tune the needed amount of redundancy ratio.

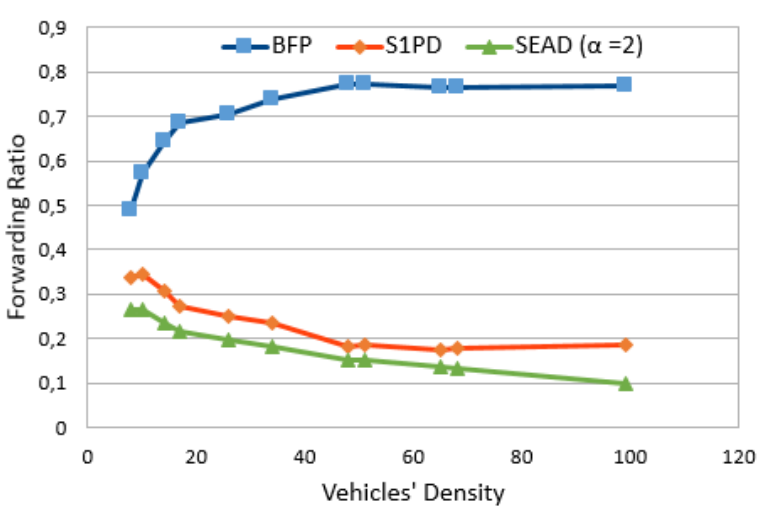

Fig. 9 Forwarding Ratio vs Various Vehicles' Density

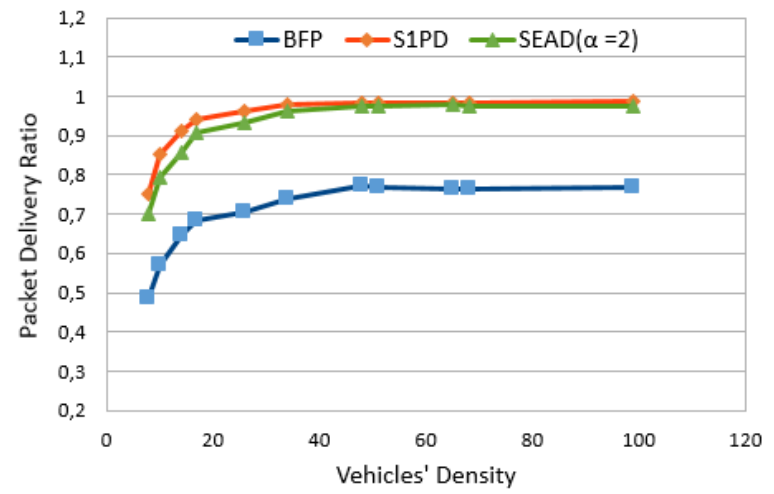

Fig. 10 Packet Delivery Ratio vs Various Vehicles' Density

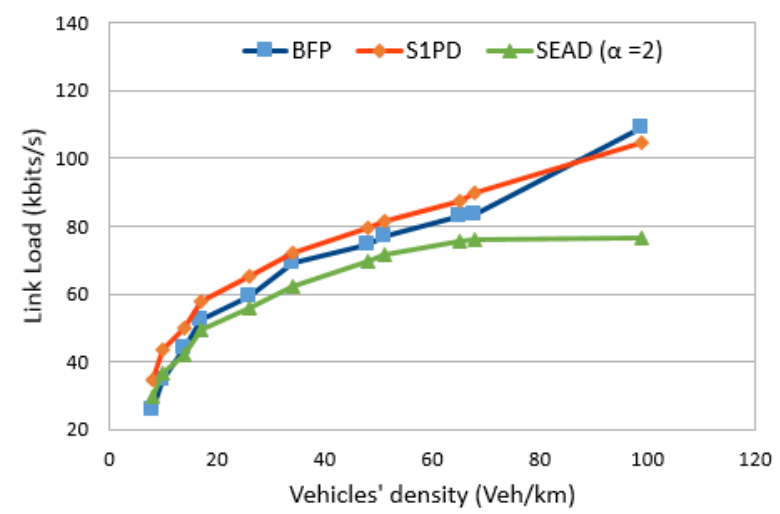

Fig. 11 Link Load vs Various Vehicles' Density

\section{Concluding Remarks}

In this work, we propose a simple and efficient data dissemination protocol called "SEAD". This protocol aims to meet the challenging problems of broadcast storm in scalable vehicular network. For this purpose, we designed a beaconless mechanism for density-awareness. Given an updated "Redundancy Ratio", each vehicle is implicitly aware of the surrounding vehicles' density. Hence, each vehicle is able to determine its forwarding opportunity at the expiry of its waiting time, calculated 


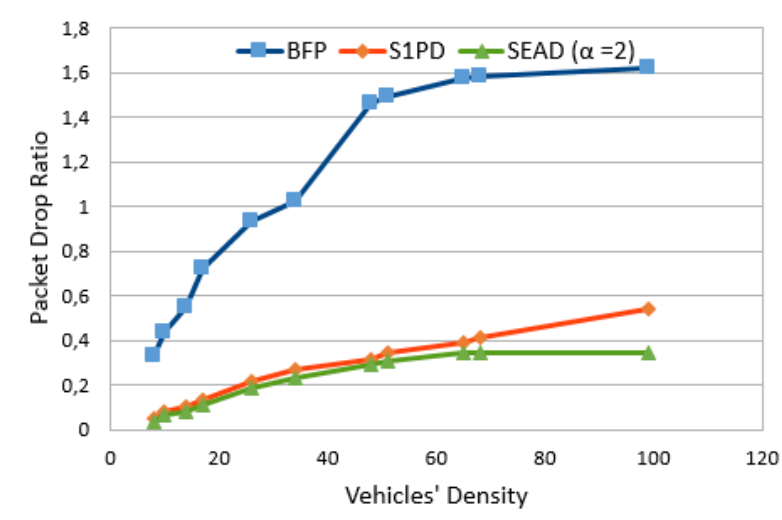

Fig. 12 Packet Drop Ratio vs Various Vehicles' Density

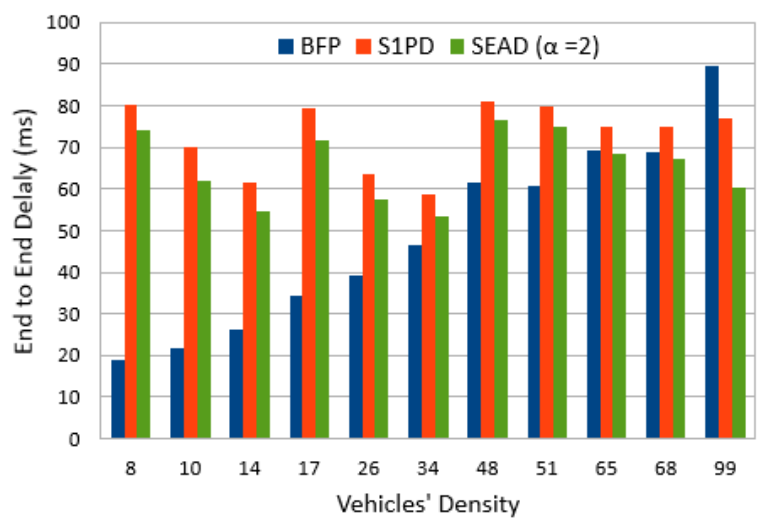

Fig. 13 End to End Delay vs Various Vehicles' Density

according to S1PD scheme. Moreover, SEAD protocol is designed to be more flexible for different types of application and to operate in adaptive mode by just tuning a particular $\alpha$ parameter.

Simulation results showed the robustness of this protocol to adapt different applications' requirements under various vehicles' density. In particular, extensive simulations demonstrated the high level performance of SEAD for safety message dissemination. Compared to S1PD, SEAD has achieved an outstanding improvement in providing a high PDR within a low end-to-end delay while optimizing the limited bandwidth consumption.

Future work includes the accommodation of SEAD to sparse networks and the investigation of the connectivity problem between communicating vehicles. Furthermore, the network resources consumption may be further saved by applying the network coding pradigm while taking into account its networking complexity.

Acknowledgements This work is a part of the MOBIDOC project achieved under the PASRI program, funded by the European Union and administrated by the ANPR.

\section{References}

1. Achour, I., Bejaoui, T., Tabbane, S.: Network coding approach for vehicle-to-vehicle communication: Principles, protocols and benefits. In: Software, Telecommunications and Computer Networks (SoftCOM), 2014 22nd International Conference on, pp. 154-159. IEEE (2014)

2. Ahlswede, R., Cai, N., Li, S.Y., Yeung, R.W.: Network information flow. Information Theory, IEEE Transactions on 46(4), 1204-1216 (2000)

3. Bhuiyan, M.M., Salim, S.M., Hasan, M.R.: Density aware broadcasting scheme for vanet. In: Communications (APCC), 2011 17th Asia-Pacific Conference on, pp. 467-471. IEEE (2011)

4. Blum, J.J., Eskandarian, A.: Avoiding timeslot boundary synchronization for multihop message broadcast in vehicular networks. In: Vehicular Technology Conference, 2009. VTC Spring 2009. IEEE 69th, pp. 1-5. IEEE (2009)

5. Chaqfeh, M., Lakas, A., Jawhar, I.: A survey on data dissemination in vehicular ad hoc networks. Vehicular Communications 1(4), 214-225 (2014)

6. Dong-Won, K., Ajmal, K., You-Ze, C.: Traffic density-based broadcast scheme for vehicular ad hoc networks. IEICE transactions on communications 95(12), 3875-3878 (2012)

7. ETSI, E.: 637-3: Its. Vehicular Communications; Basic Set of Applications; Part 2: Specification of Decentralized Environmental Notification Basic Service (2014)

8. ETSI, T.: Intelligent transport systems (its); vehicular communications; basic set of applications; definitions. Tech. rep., Tech. Rep. ETSI TR 102 $638(2009)$

9. Group, I..W., et al.: Ieee standard for information technology-telecommunications and information exchange between systems-local and metropolitan area networks-specific requirements-part 11: Wireless lan medium access control (mac) and physical layer (phy) specifications amendment 6: Wireless access in vehicular environments. IEEE Std 802, $11 \mathrm{p}(2010)$

10. Kumar, R., Dave, M., et al.: A review of various vanet data dissemination protocols. International Journal of U-and E-Service, Science and Technology (IJUNESST) 5(3), 27-44 (2012)

11. Li, P., Guo, S., Yu, S., Vasilakos, A.V.: Reliable multicast with pipelined network coding using opportunistic feeding and routing. Parallel and Distributed Systems, IEEE Transactions on 25(12), 3264-3273 (2014) 
12. Lipman, J., Liu, H., Stojmenovic, I.: Broadcast in ad hoc networks. In: Guide to Wireless Ad Hoc Networks, pp. 121-150. Springer (2009)

13. Liu, J., Wan, J., Wang, Q., Deng, P., Zhou, K., Qiao, Y.: A survey on position-based routing for vehicular ad hoc networks. Telecommunication Systems pp. 1-16 (2015)

14. Liu, J., Wan, J., Wang, Q., Li, D., Qiao, Y., Cai, H.: A novel energy-saving one-sided synchronous two-way ranging algorithm for vehicular positioning. Mobile Networks and Applications pp. 1-12 (2015)

15. Liu, L., Song, Y., Zhang, H., Ma, H., Vasilakos, A.V.: Physarum optimization: A biology-inspired algorithm for the steiner tree problem in networks. Computers, IEEE Transactions on 64(3), 819-832 (2015)

16. Liu, Y., Xiong, N., Zhao, Y., Vasilakos, A.V., Gao, J., Jia, Y.: Multi-layer clustering routing algorithm for wireless vehicular sensor networks. IET communications 4(7), 810-816 (2010)

17. Marwaha, S., Srinivasan, D., Tham, C.K., Vasilakos, A.: Evolutionary fuzzy multi-objective routing for wireless mobile ad hoc networks. In: Evolutionary Computation, 2004. CEC2004. Congress on, vol. 2, pp. 1964-1971. IEEE (2004)

18. NS3: ns-3 - network simulator project (2014). URL https://www.nsnam.org/

19. Panichpapiboon, S., Pattara-Atikom, W.: A review of information dissemination protocols for vehicular ad hoc networks. Communications Surveys \& Tutorials, IEEE 14(3), 784-798 (2012)

20. Rahimi, M.R., Venkatasubramanian, N., Mehrotra, S., Vasilakos, A.V.: Mapcloud: mobile applications on an elastic and scalable 2-tier cloud architecture. In: Proceedings of the 2012 IEEE/ACM Fifth International Conference on Utility and Cloud Computing, pp. 83-90. IEEE Computer Society (2012)

21. Schwartz, R.S., Barbosa, R.R., Meratnia, N., Heijenk, G., Scholten, H.: A directional data dissemination protocol for vehicular environments. Computer Communications 34(17), 2057-2071 (2011)

22. Schwartz, R.S., Das, K., Scholten, H., Havinga, P.: Exploiting beacons for scalable broadcast data dissemination in vanets. In: Proceedings of the ninth ACM international workshop on Vehicular internetworking, systems, and applications, pp. 53-62. ACM (2012)

23. Schwartz, R.S., Scholten, H., Havinga, P.: A scalable data dissemination protocol for both highway and urban vehicular environments. EURASIP Journal on Wireless Communications and Networking 2013(1), 1-19 (2013)
24. Spyropoulos, T., Rais, R.N., Turletti, T., Obraczka, K., Vasilakos, A.: Routing for disruption tolerant networks: taxonomy and design. Wireless networks 16(8), 2349-2370 (2010)

25. SUMO: Sumo - simulation of urban mobility (2013). URL http://sumo.sourceforge.net/

26. Tseng, Y.C., Ni, S.Y., Chen, Y.S., Sheu, J.P.: The broadcast storm problem in a mobile ad hoc network. Wireless networks 8(2-3), 153-167 (2002)

27. Tseng, Y.T., Jan, R.H., Chen, C., Wang, C.F., Li, H.H.: A vehicle-density-based forwarding scheme for emergency message broadcasts in vanets. In: Mobile Adhoc and Sensor Systems (MASS), 2010 IEEE 7th International Conference on, pp. 703708. IEEE (2010)

28. Vasilakos, A.V., Zhang, Y., Spyropoulos, T.: Delay tolerant networks: Protocols and applications. CRC press (2011)

29. Wisitpongphan, N., Tonguz, O.K., Parikh, J.S., Mudalige, P., Bai, F., Sadekar, V.: Broadcast storm mitigation techniques in vehicular ad hoc networks. Wireless Communications, IEEE 14(6), 8494 (2007)

30. Yen, Y.S., Chao, H.C., Chang, R.S., Vasilakos, A.: Flooding-limited and multi-constrained qos multicast routing based on the genetic algorithm for manets. Mathematical and Computer Modelling 53(11), 2238-2250 (2011)

31. Yeung, R.W.: Network coding: A historical perspective. Proceedings of the IEEE 99(3), 366-371 (2011)

32. Zeng, Y., Xiang, K., Li, D., Vasilakos, A.V.: Directional routing and scheduling for green vehicular delay tolerant networks. Wireless networks 19(2), 161-173 (2013)

33. Zhou, L., Xiong, N., Shu, L., Yeo, S.S., et al.: Context-aware middleware for multimedia services in heterogeneous networks. IEEE Intelligent Systems 25(2), 40-47 (2010) 